\title{
Synthesis of Poly(ethylene adipate) with a Stable Nitroxyl Radical at Both Chain Ends, and Applications to a Counter Radical for Living Radical Polymerization
}

\author{
Eri YoshIDA ${ }^{\dagger}$ and Masashi NaKamura \\ Department of Polymer Science and Engineering, Kyoto Institute of Technology. \\ Goshokaido-cho, Matsugasaki, Sakyo-ku, Kyoto 606-8585, Japan
}

(Received March 23, 1998)

\begin{abstract}
Synthesis of poly(ethylene adipate) (PEA) with 2,2,6,6-tetramethylpiperidine-1-oxyl (TEMPO) at both chain ends was attained by reaction of 4-hydroxy-TEMPO and PEA with isocyanate groups at the terminals. PEA obtained was found to have the living nitroxyl radical, because the electron spin resonance (ESR) spectrum of the polymer manifested a typical three sharp signals based on TEMPO. TEMPO-supported PEA served as a polymeric counter radical for the radical polymerization of styrene, to quantitatively give the block copolymer of ethylene adipate and styrene. Polymerization proceeded in accordance with a living mechanism, because the molecular weight of the copolymer was proportional to conversion of styrene and reciprocal of initial concentration of TEMPO attached to PEA. Analysis of the copolymer using a thin layer chromatography/flame ionization detection (TLC/FID) clarified that the copolymer contained no homopolystyrene. This absence of homopolystyrene implies that no diblock copolymer is formed during polymerization. ${ }^{1} \mathrm{H}$ NMR analysis demonstrated the structure of copolymer obtained to be A-B-A triblock.

KEY WORDS Poly(ethylene adipate) / 2,2,6,6-Tetramethylpiperidine-1-Oxyl / Living Radical Polymerization / Triblock Copolymer /
\end{abstract}

Aliphatic polyester is of medical importance as a biodegradable and biocompatible material. This polymer has been used to create materials for an artificial blood vessel and suture. The block and random copolymers containing the aliphatic polyester have been synthesized for improvement of mechanical and physical properties. Examples include the block copolymers of $\varepsilon$-caprolactone with trimethylene carbonate, lactide, ${ }^{1}$ and with adipic anhydride, ${ }^{2}$ and random copolymers of $\varepsilon$-caprolactone with its derivatives. ${ }^{3}$ It is significant to introduce functional groups into the polyester, because reactions of the groups attached to the polymer lead to create new macromolecular architecture containing the polyester. There are some papers on the synthesis of polymers with end-functional groups such as olefin, ${ }^{4}$ halogen, and amino groups. ${ }^{5}$ This functionalization was performed all for $\operatorname{poly}(\varepsilon$-caprolactone). We released a publication on the synthesis of poly( $\varepsilon$-caprolactone) with a stable nitroxyl radical at the terminal. ${ }^{6}$ The nitroxyl radical 2,2,6,6-tetramethylpiperidine-1-oxyl (TEMPO) is known as an anti-oxidant, and as a mediator for the oxidation of alcohols by copper(II). ${ }^{7}$ This radical also serves as an efficient counter radical for living radical polymerization of styrene ${ }^{8-15}$ and its derivatives, ${ }^{16-18}$ giving polymers with narrow polydispersities. We already quantitatively obtained block copolymers of styrene with $\varepsilon$-caprolactone $^{6}$ and with tetrahydrofuran (THF), ${ }^{19,20}$ by the living radical polymerization using TEMPO supported on the polymers. This paper describes the synthesis of poly(ethylene adipate) (PEA) with TEMPO at both chain ends and block copolymers of PEA and polystyrene by the radical polymerization of styrene using PEA as a counter radical. PEA is important as a biodegradable polymers obtained by polycondensation and polystyrene is significant in medical field, as a medical packaging

\footnotetext{
$\uparrow$ To whom correspondence should be addressed
}

material and as a material for disposable implements of test tubes. Their copolymers are expected to be used to create nontoxic materials for artificial internal organs which have only the desirable parts are degraded in the body: for instance, a membrane for artificial dialysis and a millipore filter.

\section{EXPERIMENTAL}

\section{Measurements}

Gel permeation chromatography (GPC) was performed with a Tosoh HLC-802A instrument equipped with a RI detector and with a Tosoh CP-8000 chromato processor. A combination of two polystyrene gel columns of Tosoh TSK gel G4000 $\mathrm{H}_{8}$ and $\mathrm{G} 2000 \mathrm{H}_{8}$ was used with THF as the eluent at $42^{\circ} \mathrm{C}$. ${ }^{1} \mathrm{H}$ NMR spectra were obtained with a Bruker ARX-500 NMR spectrometer and UV spectra were obtained with a Shimadzu UV2500PC UV-VIS recording spectrophotometer. The electron spin resonance (ESR) spectra were recorded on a JEOL JES-TE 300 ESR spectrometer. Gas chromatography (GC) was performed with a Shimadzu GC-6A. The thin layer chromatography/flame ionization detection (TLC/FID) method $^{21}$ was used with an Iatroscan TH-10 TLC analyzer.

\section{Materials}

PEA with isocyanate groups at the terminals (functionality $=2.00$ ), obtained by reaction of PEA terminal diol and tolylene 2,4-diisocyanate was supplied from Nippon Polyurethane Ind. Co., Ltd. 4-Hydroxy-TEMPO was prepared by the method reported previously. ${ }^{22} \mathrm{THF}$ was purified by refluxing on sodium for several hours and distilled over sodium. Triethylamine was purified by refluxing on calcium hydride for several hours and distilled. Commercial grade styrene was washed with an aqueous alkaline solution and water, and distilled over 


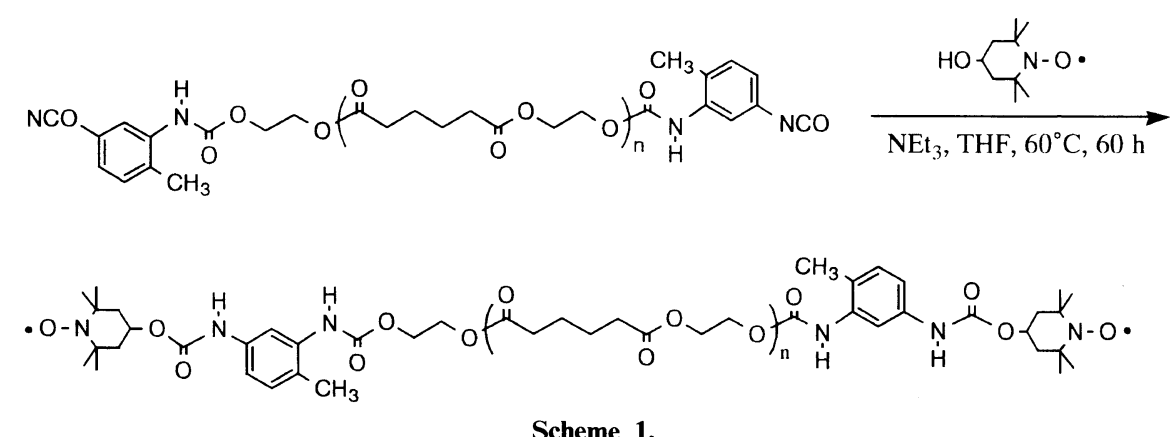

Scheme 1.

calcium hydride. Benzoyl peroxide (BPO) was precipitated from chloroform into methanol and then recrystallized at $0^{\circ} \mathrm{C}$. Extrapure grade phenylhydrazine for ${ }^{1} \mathrm{H}$ NMR studies was used without further purification.

\section{Reaction of 4-Hydroxy-TEMPO and PEA with Isocya- nate Groups at the Terminals}

PEA with isocyanate groups at the terminals $(3.00 \mathrm{~g}$, containing $2.99 \mathrm{mmol}$ isocyanate group) was dissolved in THF of $20 \mathrm{~mL}$. To the solution 4-hydroxy-TEMPO $(1.50 \mathrm{~g}, 8.71 \mathrm{mmol})$ and triethylamine $(0.1 \mathrm{~mL}, 0.717$ mmol) were added. The mixture was kept at $60^{\circ} \mathrm{C}$ for $60 \mathrm{~h}$. The solution was evaporated and dried in vacuo to remove THF and triethylamine. The crude product was reprecipitated from THF into hexane three times, and dried in vacuo for several hours to yield $3.23 \mathrm{~g}$ of the polymer.

\section{Radical Polymerization of Styrene by BPO and PEA with TEMPO at Both the Chain Ends}

Styrene (1.0 mL, $8.73 \mathrm{mmol})$, BPO (11.1 mg, 0.0458 mmol), and PEA $(68.1 \mathrm{mg}$, containing $0.0620 \mathrm{mmol}$ of TEMPO moiety) were placed in an ampule. After degassing, the ampule was sealed in vacuo. Polymerization was carried out at first for $3.5 \mathrm{~h}$ at $95^{\circ} \mathrm{C}$, and continued for another $48 \mathrm{~h}$ at $125^{\circ} \mathrm{C}$. It was terminated by cooling with liquid nitrogen. The product was dissolved in $10 \mathrm{~mL}$ dichloromethane, purified by repeated precipitations from dichloromethane into hexane, and finally dried in vacuo for several hours. The block copolymer $(711 \mathrm{mg})$, was obtained. After the addition of toluene as an internal standard, the dichloromethane solution was subjected to GC to estimate the conversion of styrene.

\section{RESULTS AND DISCUSSION}

PEA with isocyanate groups at the terminals was obtained by reaction of PEA terminal diol and tolylene 2,4-diisocyanate (TDI). The molecular weight of the PEA with diisocyanate was estimated as 2010 , by ${ }^{1} \mathrm{H}$ NMR based on the relative intensity of the methylene protons of the ethylene glycol units and the methyl ones of the TDI. The molecular weight and polydispersity of this PEA were 2000 and 1.75 respectively, determined by GPC calibrated with polystyrene standards. Reaction of the PEA and 4-hydroxy-TEMPO was performed in THF at $60^{\circ} \mathrm{C}$ for $60 \mathrm{~h}$, in the presence of triethylamine (Scheme 1). Absorbance at $2270 \mathrm{~cm}^{-1}$ based on the isocyanate

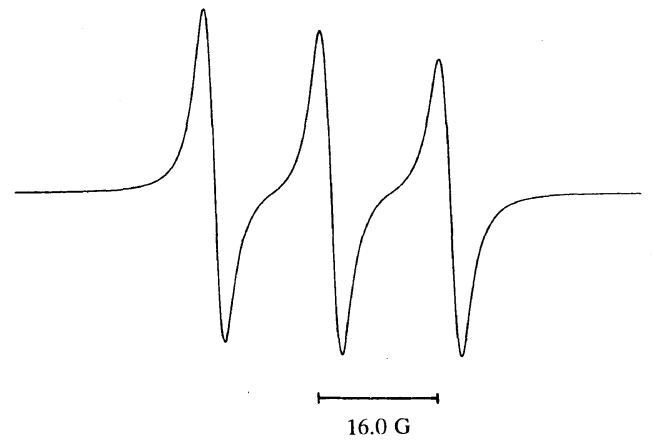

Figure 1. ESR spectrum of PEA obtained by reaction of 4-hydroxyTEMPO and PEA with isocyanate groups at the terminals (solvent: benzene, at room temperature).

disappeared completely in IR, after the reaction. GPC of the product shifted to the higher molecular weight side, and gave molecular weight and polydispersity as 3400 and 1.86 , respectively. The resulting polymer, isolated and purified by repeated reprecipitation, was highly viscous and orange, suggesting the presence of the living nitroxyl radical. The ESR spectrum of the polymer demonstrated the presence of TEMPO attached to PEA, because the polymer manifested typical three sharp signals based on TEMPO (Figure 1). $g$ and hyperfine coupling constant of the polymer $\left(g=2.00716, A_{\mathrm{N}}=\right.$ $16.0 \mathrm{G})$ were identical to those of 4-methoxy-TEMPO $\left(g=2.00717, A_{\mathrm{N}}=16.0 \mathrm{G}\right)$. The ${ }^{1} \mathrm{H}$ NMR spectrum of the resulting polymer also demonstrated the presence of TEMPO. The spectrum is shown in Figure 2. Signals at 6.8 and $7.2 \mathrm{ppm}$ originate from phenylhydrazine, added for reducing the TEMPO moiety into the corresponding hydroxylamine, for ${ }^{1} \mathrm{H}$ NMR study. Signals at 4.15, 2.36, and $1.66 \mathrm{ppm}$ were attributed to PEA. Signals at 4.15 ppm originate from the methylene protons of the ethylene glycol units. Signals at $2.36 \mathrm{ppm}$ were assigned to the methylene protons attached to the carbonyl carbon, and those at $1.66 \mathrm{ppm}$ to another methylene bonded to this methylene. Signals were seen at $7.06 \mathrm{ppm}$, and assigned to the aromatic protons of the TDI moieties attached to both chain ends of PEA. These protons were located at 5 and 6 positions of the aromatic ring. The signal at $2.18 \mathrm{ppm}$ was due to the TDI, and assigned to the methyl proton bonded to the aromatic ring. Signals originating from TEMPO were at 1.24 (a), 1.26 (b), 1.84 (c), and $2.02 \mathrm{ppm}(\mathrm{d})$ and assigned to tetramethyl protons, axial (a) and equatorial (b), and methylene ones, axial (c) and equatorial (d), respectively. Signals at $5.04 \mathrm{ppm}$ were attributed to methine protons of TEMPO. The degree of introduction (DI) of TEMPO into PEA was estimat- 


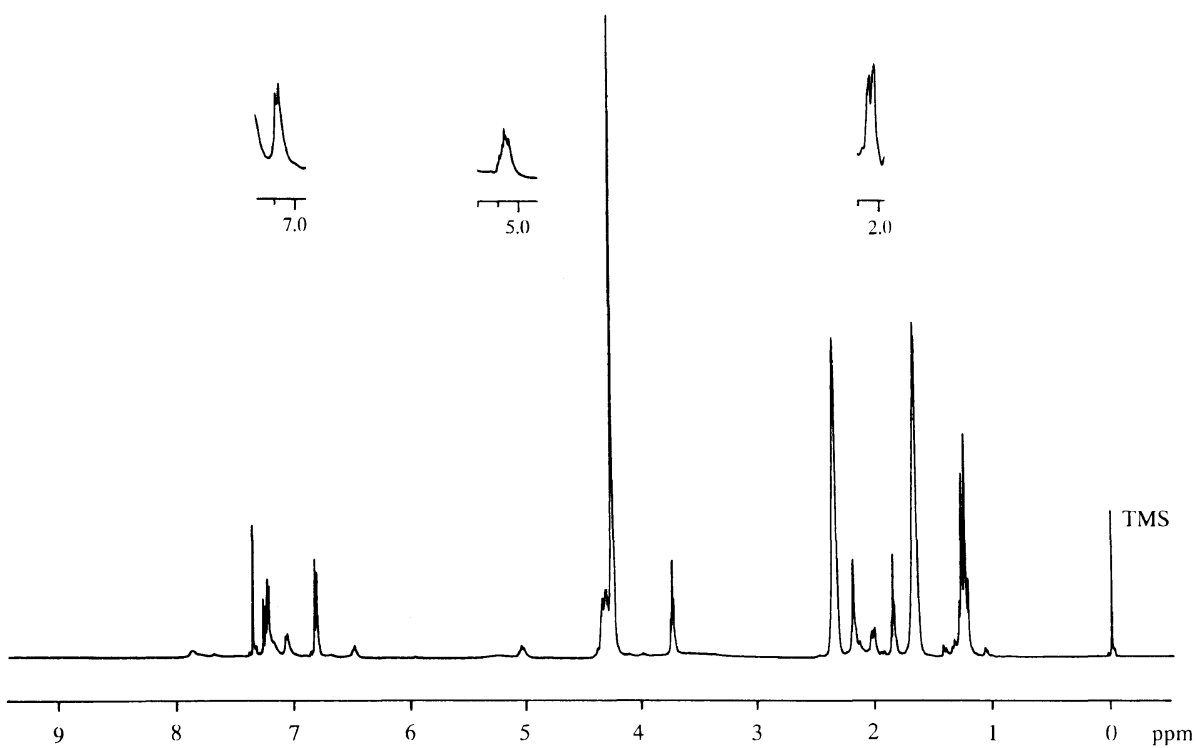

Figure 2. ${ }^{1} \mathrm{H}$ NMR spectrum of PEA obtained by reaction of 4-hydroxy-TEMPO and PEA with TDI moieties at both chain ends (solvent: $\mathrm{CDCl}_{3}$, in the presence of phenylhydrazine).

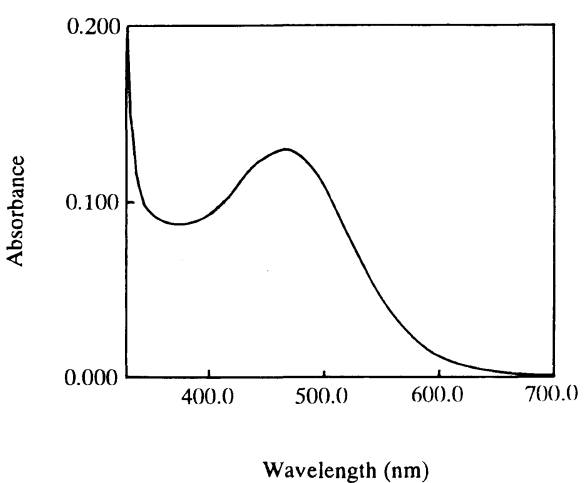

Figure 3. UV spectrum of PEA (solvent: THF).

ed to be 0.929 , from relative intensity of the methyl protons of the TDI and tetramethyl ones of TEMPO. The molecular weight of the resulting polymer was estimated to be 2100 , by ${ }^{1} \mathrm{H}$ NMR using the intensity of the methylene protons of the ethylene glycol units and the methyl ones of the TDI. It was assumed that TEMPO was quantitatively introduced into both chain ends of PEA, and the molecular weight was estimated to be 2210 , by ${ }^{1} \mathrm{H}$ NMR based on the intensity of methylene protons of the ethylene glycol units and tetramethyl ones of TEMPO. DI and molecular weight were calculated from UV analysis. The UV spectrum of PEA obtained is shown in Figure 3. PEA had characteristic UV absorption $\lambda_{\max }$ at $465 \mathrm{~nm}$, originating from the nitroxyl radical. DI was estimated as 1.07 , based on absorbance intensity at this wavelength and on the theoretical molecular weight of PEA. This molecular weight was 2350 , calculated using the molecular weight $\left(M_{n}=2010\right)$ of PEA with isocyanate groups at the terminals. The molecular weight of the resulting polymer was 2200 , from the content of TEMPO per gram of the polymer. The content, determined by UV was $0.910 \mathrm{mmol} \mathrm{g}^{-1}$. The four molecular weights of 2350, 2100, 2210, and 2200 were in close agreement. We deduced that TEMPO was quantitatively introduced into the chain ends of PEA, and therefore, the synthesis of PEA with TEMPO at both chain ends was attained.

Radical polymerization of styrene was performed by BPO as an initiator using PEA obtained. Polymerization was carried out in bulk at $125^{\circ} \mathrm{C}$ for $48 \mathrm{~h}$, after being held at $95^{\circ} \mathrm{C}$ for $3.5 \mathrm{~h}$ (Scheme 2). The rate of polymerization was much faster with TEMPO supported on polymers, than that with TEMPO of low molecular weight, such as 4-methoxy-TEMPO. ${ }^{6,19,20}$ Therefore, the two-step procedure of heating was used to give block copolymers with narrow polydispersities, by dividing the initiation from the propagation. ${ }^{8}$ It is also expected that uncontrolled-polymerization of styrene proceeds more easily in polymerization by TEMPO-supported polymers than that by low-molecular weight TEMPO, because mobility of TEMPO is restrained by PEA chain attached to TEMPO. Excess TEMPO over BPO was used for the polymerization to check the uncontrolled-polymerization. This is because TEMPO controls polymerization initiated not only by BPO, but also by thermal initiator. ${ }^{24}$ The conversion of styrene was calculated to be $91 \%$ by GC. GPCs of the copolymer and of PEA as a prepolymer are shown in Figure 4. GPC of the resulting copolymer was higher and exhibited a unimodal curve. Molecular weight and polydispersity were 33100 and 1.37, respectively. No peak of the prepolymer was observed in GPC of the copolymer, indicating that the copolymer was quantitatively formed. The copolymer contained no homopolymer of styrene, based on TLC/FID analysis. TLC/FIDs of the copolymer and homopolystyrene showed $\mathrm{Rf}$ as 0.022 and 0.15 , respectively. As shown in Figure 5, no peak from the homopolystyrene was observed in TLC/FID of the copolymer. This implies that undesirable reactions such as uncontrolled-polymerization of styrene and disproportionation of the aminoxy moieties do not occur during polymerization. This indicates that no diblock copolymer is generated, because the diblock copolymer is always formed with homopolystyrene by the disproportionation (Scheme 3). These results have been observed in the polymerization of styrene using TEMPO-biterminated poly(THF). ${ }^{20}$ The disproportionation of the aminoxy moiety occurs at 


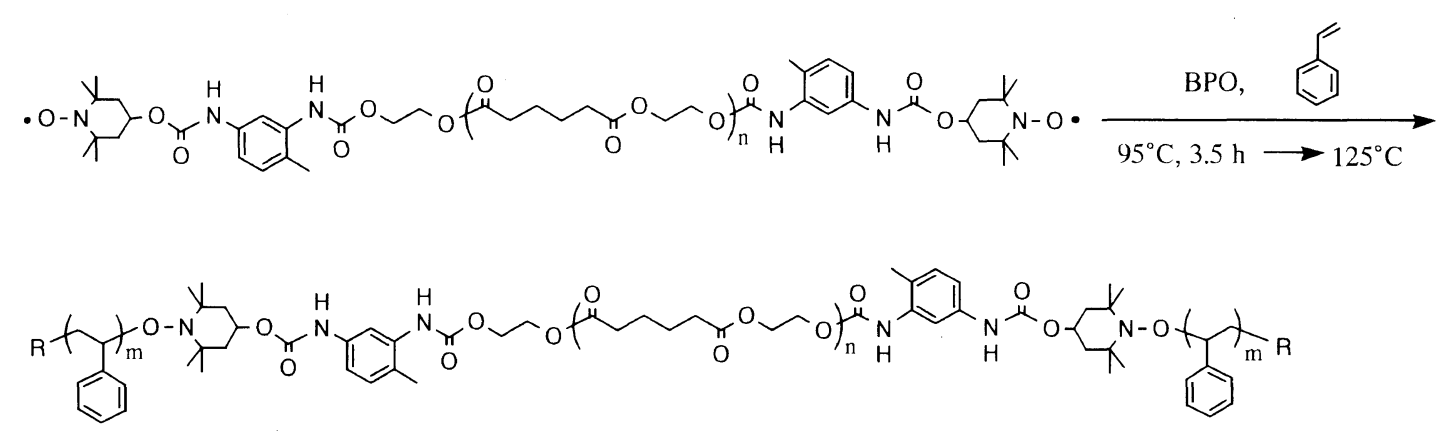

Scheme 2.

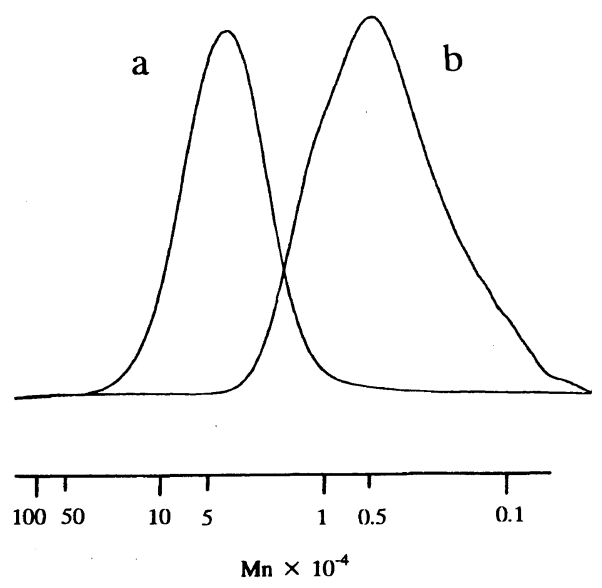

Figure 4. GPC profiles of the block copolymer (a) and prepolymer (b).

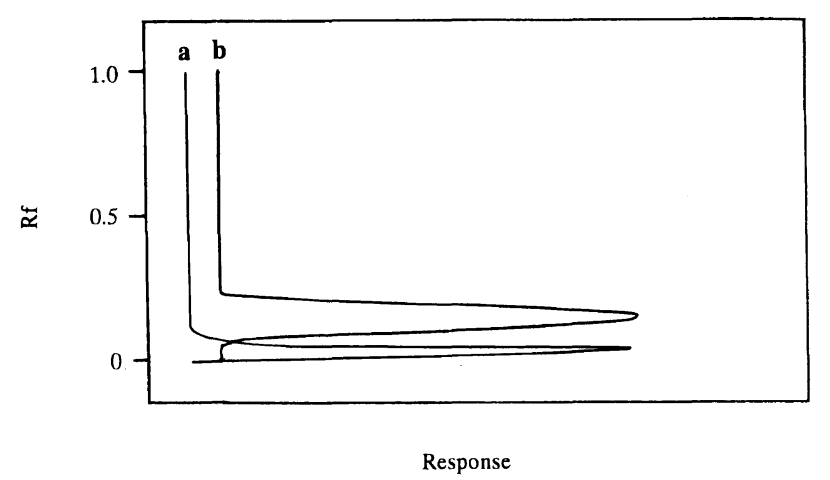

Figure 5. TLC/FID (benzene/cyclohexane $=2 / 1$ ) of the block copoly$\operatorname{mer}\left(M_{n}=33100, M_{w} / M_{n}=1.37\right)(\mathrm{a}, R f=0.022)$, and homopolystyrene $\left(M_{n}=37200\right)(\mathrm{b}, R f=0.15)$.
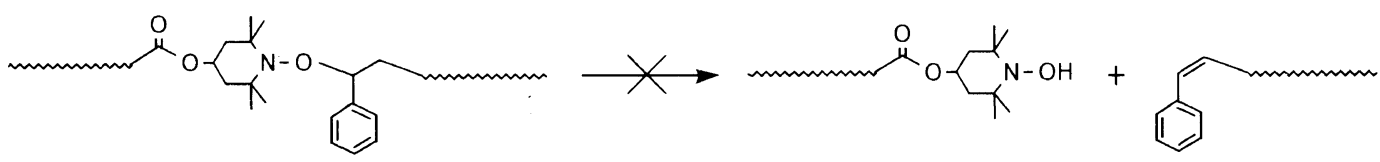

Scheme 3.

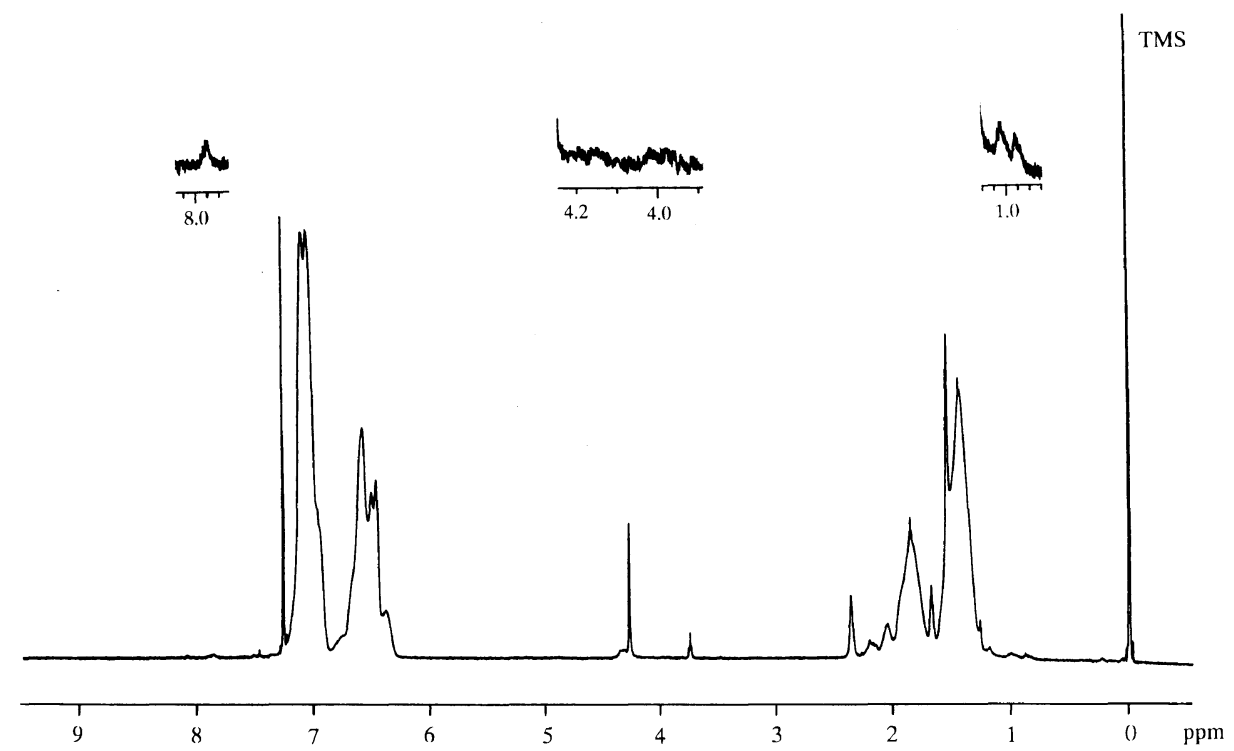

Figure 6. ${ }^{1} \mathrm{H}$ NMR spectrum of the copolymer (conversion $=28 \%, M_{n}=17300, M_{w} / M_{n}=1.51$ ), obtained by radical polymerization of styrene by BPO using TEMPO-supported PEA. Polymerization was carried out at $125^{\circ} \mathrm{C}$ for $10 \mathrm{~h}$, after being held at $95^{\circ} \mathrm{C}$ for $3.5 \mathrm{~h}$ (solvent: $\mathrm{CDCl}_{3}$ ). 


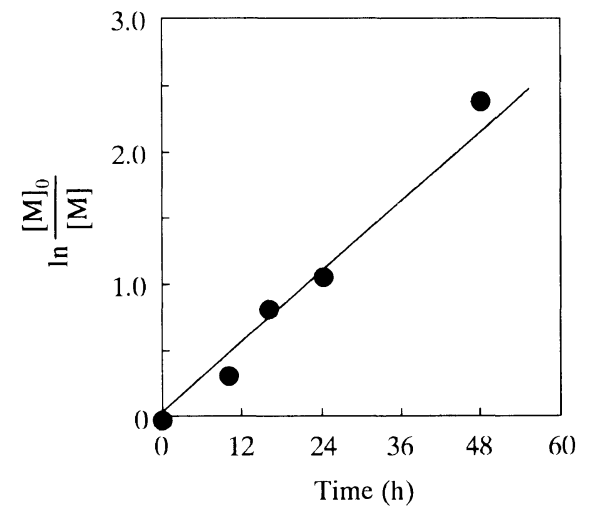

Figure 7. First order time-conversion plots in polymerization of styrene by BPO using PEA with TEMPO at both chain ends.

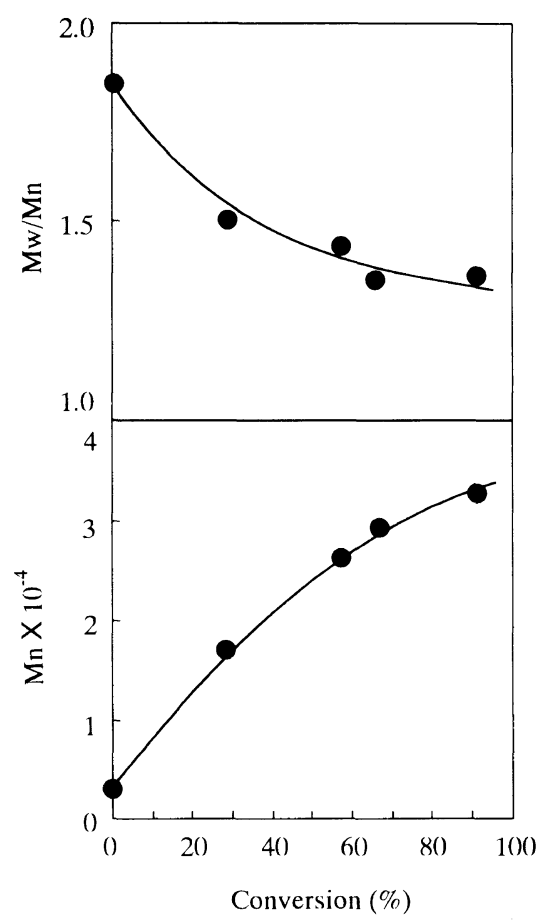

Figure 8. Plots of molecular weight and polydispersity index versus conversion in polymerization of styrene by BPO using PEA with TEMPO at both chain ends.

a later stage of the polymerization, where the monomer is entirely consumed, or exists in small amount. ${ }^{20,23}$ In fact, the disproportionation hardly proceeds in a benzene solution. ${ }^{20}$ The reason for the absence of the disproportionation during the polymerization using TEMPObiterminated PEA may be that a certain amount of styrene remains in the system at a later stage, when the conversion is taken into consideration.

${ }^{1} \mathrm{H}$ NMR analysis clarified that the structure of copolymer obtained was A-B-A triblock. The ${ }^{1} \mathrm{H}$ NMR spectrum of the copolymer $\left(M_{n}=17300\right.$, and $M_{w} / M_{n}=$ 1.51 ) is shown in Figure 6. Signals at 4.30, 2.40, and $1.70 \mathrm{ppm}$ were attributed to PEA. Signals of the aromatic protons due to the styrene units were observed at $6.2-7.6 \mathrm{ppm}$, and thus those of main chains were observed at $1.3-2.3 \mathrm{ppm}$. Signals due to the benzoyl groups were at $7.90 \mathrm{ppm}$, and assigned to aromatic protons at ortho positions. Signals were also observed at 0.90 and $1.05 \mathrm{ppm}$, and attributed to the tetramethyl protons of TEMPO combining two segments of poly-
Table I. Radical polymerization of styrene by BPO and PEA with TEMPO at both chain ends

\begin{tabular}{|c|c|c|c|c|}
\hline$[\text { TEMPO-PEA }]_{0}{ }^{a}$ & Time & Conversion $^{\mathrm{b}}$ & & \\
\hline$\times 10^{3} \mathrm{~mol} \mathrm{~L}^{-1}$ & $\mathrm{~h}$ & $\%$ & & \\
\hline 92.7 & 92 & 93 & 25400 & 1.38 \\
\hline 61.9 & 85 & 91 & 33700 & 1.46 \\
\hline 46.7 & 92 & 91 & 50700 & 1.45 \\
\hline 31.2 & 40 & 88 & 67000 & 1.54 \\
\hline
\end{tabular}

${ }^{\mathrm{a}}[\mathrm{TEMPO}-\mathrm{PEA}]_{0} /[\mathrm{BPO}]_{0}=1.3$, in all cases. ${ }^{\mathrm{b}}$ Calculated by GC. c Estimated by GPC based on polystyrene standards.

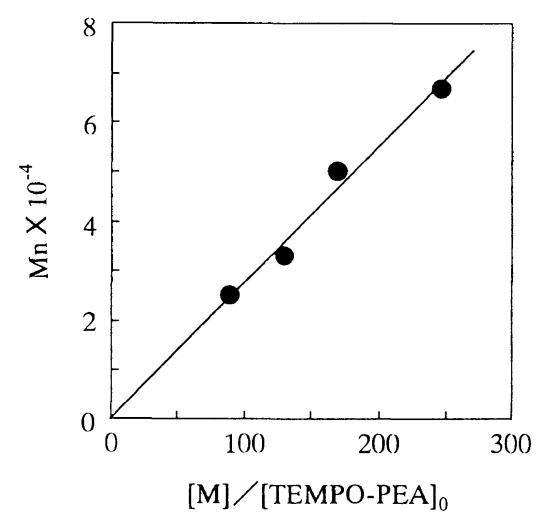

Figure 9. Plots of molecular weight versus $[\mathrm{M}] /[\text { TEMPO-PEA }]_{0}$ in polymerization of styrene by BPO using PEA with TEMPO at both chain ends.

styrene and of PEA. Signals were at $3.9-4.2 \mathrm{ppm}$, originating from terminal methine protons of polystyrene segments. This methine was bonded to the TEMPO moiety. It was assumed that all TEMPO moieties were bonded to the polystyrene segments, and the molecular weight of PEA with TEMPO at both chain ends was estimated as 2300 , based on the integral intensity of this methine protons and the methylene ones at $4.3 \mathrm{ppm}$ due to the ethylene glycol units. This molecular weight was in close agreement with the theoretical value of 2350 . Therefore, it can be concluded that the structure of the copolymer obtained was A-B-A triblock.

The radical polymerization was suggested to proceed in accordance with a living mechanism, because the first order time-conversion plots linearly increased, as demonstrated in Figure 7. Whereas the molecular weight was in almost direct proportion to the conversion of styrene, polydispersity decreased with increase of conversion (Figure 8). The concentration of the growing polymer chain is identical to the initial concentration of TEMPO. ${ }^{24}$ The relation between molecular weight and initial concentration of TEMPO supported on PEA ([TEMPO-PEA $]_{0}$ ) was investigated. The results are listed in Table I. Conversions were high enough to compare molecular weights. Molecular weight decreased as a result of increasing [TEMPO-PEA] $]_{0}$. Figure 9 demonstrates that molecular weight is directly proportional to the reciprocal of $[\text { TEMPO-PEA }]_{0}$, indicating that polymerization undoubtedly proceeds by a living mechanism.

\section{CONCLUSIONS}

The synthesis of PEA with TEMPO at both chain ends 
was attained by reaction of 4-hydroxy-TEMPO and the PEA with isocyanate groups at the terminals. The TEMPO-supported PEA served as a polymeric counter radical for the polymerization of styrene, giving the block copolymer of ethylene adipate and styrene. The copolymer was quantitatively formed, because no peak originating from the prepolymer of PEA was observed in GPC of the product. TLC/FID clarified that the resulting copolymer contained no homopolystyrene. This implies that no diblock copolymer is formed during polymerization. ${ }^{1} \mathrm{H}$ NMR analysis demonstrated the structure of copolymer to be $\mathrm{A}-\mathrm{B}-\mathrm{A}$ triblock. The radical polymerization of styrene by PEA with TEMPO at both chain ends proceeded in accordance with a living mechanism, giving poly(styrene- $b$-EA-b-styrene). This is because the molecular weight of the copolymer was in proportion to conversion of styrene and reciprocal of initial concentration of TEMPO. 4-Hydroxy-TEMPO may thus be regarded as a transforming agent from polycondensation to living radical polymerization, when it is taken into account that this compound combines two types of polymer segments obtained from polycondensation and from the living radical polymerization. This copolymer should serve as a new material that has the biodegradable polymer block as a middle segment in the triblock.

\section{REFERENCES AND NOTES}

1. Y. Shen, Z. Shen, Y. Zhang, and K. Yao, Macromolecules, 29, 8289 (1996).

2. N. Ropson, Ph. Dubois, R. Jerome, and Ph. Teyssie, J. Polym.
Sci., Polym. Chem. Ed., 35, 183 (1997).

3. J. Vion, R. Jerome, Ph. Teyssie, M. Aubin, and R. E. Prud'homme, Macromolecules, 19, 1828 (1986).

4. N. Ropson, Ph. Dubois, R. Jerome, and Ph. Teyssie, Macromolecules, 24, 977 (1991).

5. Ph. Dubois, R. Jerome, and Ph. Teyssie, Polym. Bull., 22, 475 (1989).

6. E. Yoshida and Y. Osagawa, Macromolecules, 31, 1446 (1998).

7. E. Yoshida, T. Takata, and T. Endo, Macromolecules, 25, 7282 (1992).

8. M. K. Georges, R. P. N. Veregin, P. M. Kazmaier, and G. K. Hamer, Macromolecules, 26, 2987 (1993).

9. C. J. Hawker, J. Am. Chem. Soc., 116, 11185 (1994).

10. G. Moad and E. Rizzardo, Macromolecules, 28, 8722 (1995).

11. R. P. N. Veregin, P. G. Odell, L. M. Michalak, and M. K. Georges, Macromolecules, 29, 2746 (1996).

12. K. Matyjaszewski, T. Shigemoto, J. M. J. Frechet, and M. Leduc, Macromolecules, 29, 4167 (1996).

13. T. Fukuda, T. Terauchi, A. Goto, K. Ohno, Y. Tsujii, and T. Miyamoto, Macromolecules, 29, 6393 (1996)

14. E. Yoshida and S. Tanimoto, Macromolecules, 30, 4018 (1997).

15. E. Yoshida and Y. Okada, Bull. Chem. Soc. Jpn., 70, 275 (1997).

16. E. Yoshida, J. Polym. Sci., Polym. Chem. Ed., 34, 2937 (1996).

17. E. Yoshida and T. Fujii, J. Polym. Sci., Polym. Chem. Ed., 35, 2371 (1997).

18. E. Yoshida and T. Fujii, J. Polym. Sci., Polym. Chem. Ed., 36, 269 (1998).

19. E. Yoshida and A. Sugita, Macromolecules, 29. 6422 (1996).

20. E. Yoshida and A. Sugita, J. Polym. Sci., Polym. Chem. Ed., 36, 2059 (1998).

21. E. Yoshida, T. Ishizone, A. Hirao, S. Nakahama, T. Takata, and T. Endo, Macromolecules, 27, 3119 (1994).

22. T. Kurosaki, K. W. Lee, and M. Okawara, J. Polym. Sci., Polym. Chem., 10, 3295 (1972).

23. I. Li, B. A. Howell, K. Matyjaszewski, T. Shigemoto, P. B. Smith, and D. B. Priddy, Macromolecules, 28, 6692 (1995).

24. E. Yoshida and Y. Okada, J. Polym. Sci., Polym. Chem. Ed., 34, 3631 (1996). 